\title{
Photoluminescence properties of Si nanocrystals near rough Au films
}

\author{
Nobuyuki Ishikura, Minoru Fujii , Masaki Inui and Shinji Hayashi \\ Department of Electrical and Electronic Engineering \\ Graduate School of Engineering, Kobe University, Rokkodai, Nada, Kobe 657-8501 \\ Fax: 078-803-6081, e-mail: fujii@eedept.kobe-u.ac.jp ${ }^{*}$
}

\begin{abstract}
We studied metal-enhanced photoluminescence (PL) from Si nanocrystals (Si-ncs) placed near rough $\mathrm{Au}$ films. Au films with different degree of roughness were prepared by electroless $\mathrm{Au}$ plating. We found that the PL intensity and the decay rate increase as the roughness becomes large. The PL excitation spectra revealed that the PL enhancement is the largest when the excitation wavelength corresponds to that of the surface plasmon resonance of rough $\mathrm{Au}$ films. The result combined with the emission wavelength dependence of PL enhancement factors suggest that the PL enhancement is caused by the enhancement of an electric field of incident light due to the excitation of surface plasmons supported by the rough $\mathrm{Au}$ films and also the increase of radiative decay rate of Si-ncs.

Key words: si nanocrystals, photoluminescence, surface plasmon
\end{abstract}

\section{INTRODUCTION}

Silicon nanocrystals (Si-ncs) show photoluminescence (PL) at room temperature due to the recombination of excitons confined in a zero-dimensional quantum confined system [1-4]. Since excitons are confined in a space smaller than the exciton Bohr radius of bulk Si crystals, the overlap of electron and hole wave functions becomes large in both real and momentum spaces, resulting in the enhancement of the radiative recombination rate $[2,3]$. However, even for Si-ncs as small as a few nanometers in diameter, the indirect band gap nature of bulk Si crystals is strongly inherited [5], and thus the spontaneous emission rate is still not large enough compared to that of the direct band gap semiconductors and the luminescence quantum efficiency is rather low $[2,3]$. Therefore, the enhancement of luminescence efficiency and the spontaneous emission rate of Si-ncs is in high demand for its application in optoelectric devices.

It has been demonstrated that the luminescence intensity of emitters is strongly enhanced near metal nanostructures [6-9]. The enhancement is caused by the excitation of surface plasmon polaritons (SPPs) supported by metal nanostructures. The enhancement mechanism can be classified into two processes, i.e. the enhancement of an electric field of incident light and the enhancement of the radiative decay rate. In fact, enhanced luminescence efficiency of Si-ncs was achieved by placing a nanoporous Au layer [10], a Ag island arry [7] and a Au thin film with a two-dimensional grating [11] near Si-nes.

The purpose of this work is to realize the enhancement of PL from Si-ncs by rough Au films and to investigate the correlation of the PL enhancement factor with the degree of roughness of Au films. From the emission wavelength and the excitation wavelength dependences of PL enhancement factors, we conclude that the PL enhancement is caused by the enhancement of an electric field of incident light due to the excitation of surface plasmons supported by the rough $\mathrm{Au}$ films and also the increase of radiative decay rate of Si-ncs.

\section{EXPERIMENTAL PROCEDURE}

$\mathrm{SiO}_{2}$ films containing Si-ncs (Si-ne: $\mathrm{SiO}_{2}$ films) were prepared by a cosputtering method $[12,13]$. Si and $\mathrm{SiO}_{2}$ were simultaneously sputter deposited on a $\mathrm{SiO}_{2}$ substrate, and the deposited films (150 $\mathrm{nm}$ in thickness) were annealed in a nitrogen gas atmosphere for $30 \mathrm{~min}$ at $1150{ }^{\circ} \mathrm{C}$. Si-ncs were grown in the films during the annealing. After the annealing, Si-nc: $\mathrm{SiO}_{2}$ films were etched from the top by reactive ion etching with $\mathrm{CHF}_{3}$ (L-201D, ANELVA) and the thickness is decreased to $125 \mathrm{~nm}$. On the Si-nc:SiO 2 film, a Au film $5 \mathrm{~nm}$ in thickness was deposited by vacuum evaporation. Samples were then exposed to electroless $\mathrm{Au}$ plating solution $\left(80^{\circ} \mathrm{C}\right)$ obtained from aqueous solution of 0.01 $\mathrm{mM} \mathrm{HAuCl}_{4}$ (Wako) and $0.2 \mathrm{mM} \mathrm{2-Aminoethanethiol}$ (Wako). The exposure durations were $0,0.5,1,1.5$ and 2 min. The morphology of the sample surface was studied by atomic force microscopy (AFM). Absorption spectra of the Au films were measured by a UV-visible-NIR scanning spectrophotometer (UV-3101PC, Shimadzu).

PL measurements were performed by using a single monochromator equipped with a liquid $\mathrm{N}_{2}$ cooled InGaAs near infrared diode array. The excitation source was a $457.9 \mathrm{~nm}$ line of an Ar ion laser. The excitation and the detection of PL were made through a transparent fused quartz substrate. The spectral response of the detection system was calibrated with the aid of a reference spectrum of a standard tungsten lamp. For the measurement of PL decay dynamics and photoluminescence excitation (PLE) spectra, samples were excited by pulsed light from an optical parametric oscillator (OPO) pumped by the third harmonic of a $\mathrm{Nd}$ YAG laser through the transparent fused quartz substrates. The pulse width and the repetition frequency were $5 \mathrm{~ns}$ and $20 \mathrm{~Hz}$, respectively. PL decay signals were detected by a near-infrared photomultiplier 


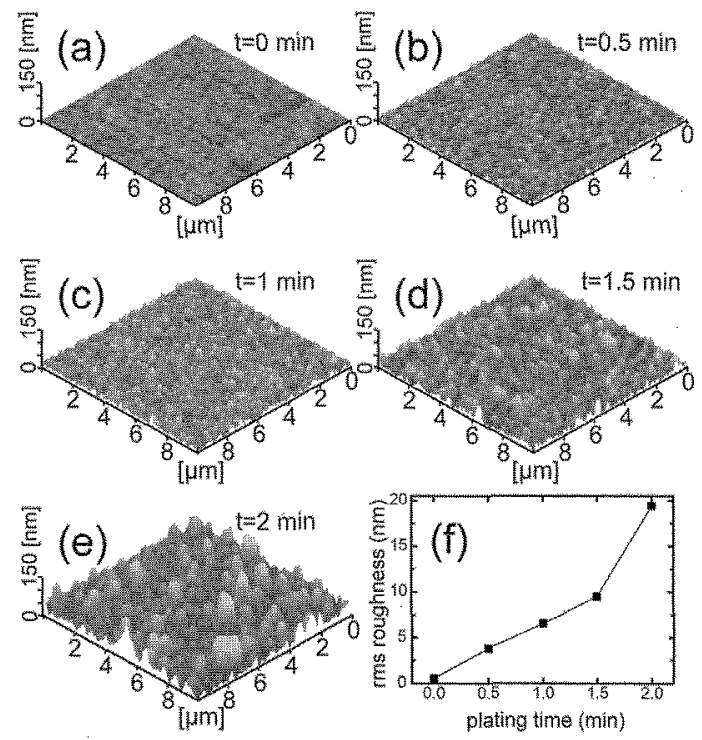

Fig. 1. AFM images of rough Au films; the plating times are (a) 0 (b) 0.5 (c) 1 (d) 1.5 and (e) $2 \mathrm{~min}$. (f) Variations of rms roughness of rough $\mathrm{Au}$ films as a function of plating times.

(R5509-72, Hamamatsu) and PLE signals were detected by photomultiplier (R943-02, Hamamatsu). The decay curves and PLE spectra were recorded by a multichannel scalar (SR430, Stanford Research). All measurements were performed at room temperature.

\section{RESULTS AND DISCUSSION}

Figures 1(a)-(e) show AFM images of sample surface. The plating time are changed from 0 to $2 \mathrm{~min}$. The roughness of $\mathrm{Au}$ films increases as the plating time increases; the root mean square (rms) roughness values are (a) 0.56 (b) 3.85 (c) 6.56 (d) 9.49 and (e) $19.41 \mathrm{~nm}$ (see Fig. 1(f)). The absorption spectra are shown in Fig. 2. The broad absorption bands due to surface plasmon resonance of the rough $\mathrm{Au}$ film can be seen. The absorption band shifts to longer wavelength and broadens as the plating time increases.

The PL spectra are shown in Fig. 3(a). The PL band covering the 700-1100 $\mathrm{nm}$ range arises from Si-ncs. We can see that the PL intensity increases as the plating time

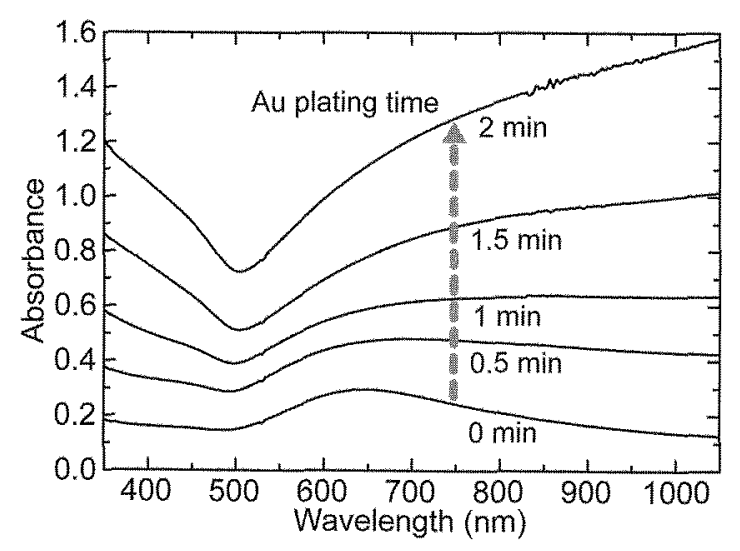

Fig. 2. Absorption spectra of rough Au films after plating different period. The plating time are changed from 0 to $2 \mathrm{~min}$.

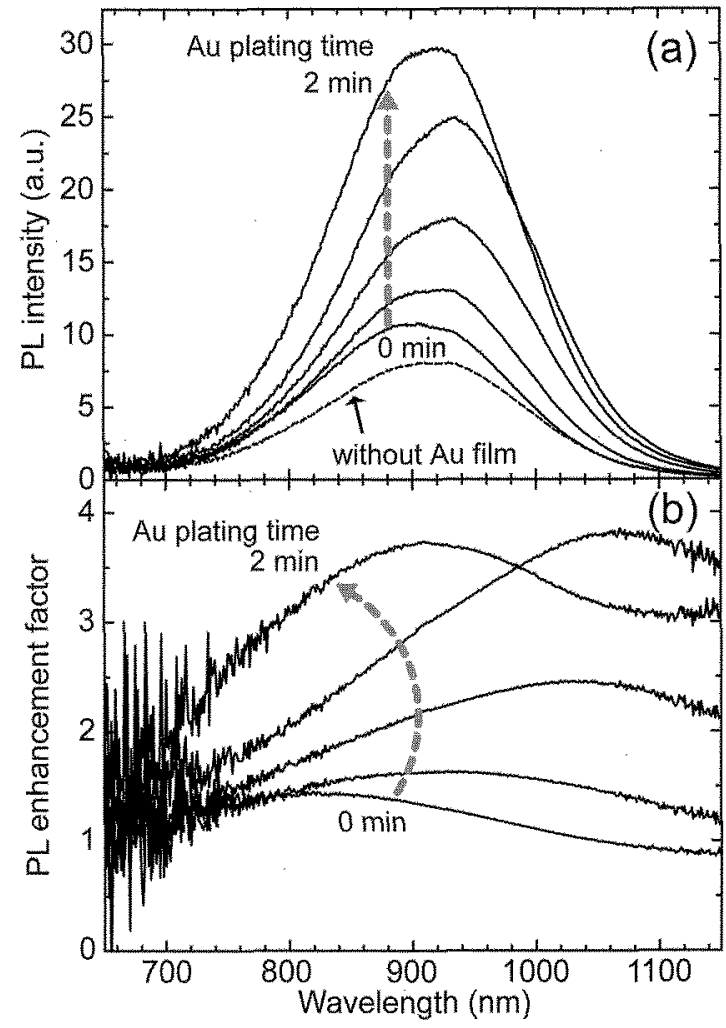

Fig. 3. (a) PL spectra of the sample with the rough $\mathrm{Au}$ film (solid line) and that without rough $\mathrm{Au}$ film (dotted line). (b) Wavelength dependence of PL enhancement factors.

increases. At the 2 minute of plating, a 3.7 -fold enhancement of the peak PL intensity is observed. Figure 3(b) shows the wavelength dependence of enhancement factors obtained by dividing the PL spectrum of the sample with a Au film by that without a $\mathrm{Au}$ film. The enhancement factors depend on the wavelength. The enhancement factor maximum shifts from 812 to $1068 \mathrm{~nm}$ with the increase of the plating time from 0 to $1.5 \mathrm{~min}$ and then shifts to shorter wavelength $(908 \mathrm{~nm})$ after 2 minute plating.

To study the mechanism of the PL enhancement, we measured the PL decay curves of the samples with a rough $\mathrm{Au}$ film at $960 \mathrm{~nm}$ (Fig. 4). The excitation wavelength is $457.9 \mathrm{~mm}$ and the decay curves are normalized at the maximum intensities. The lifetimes are

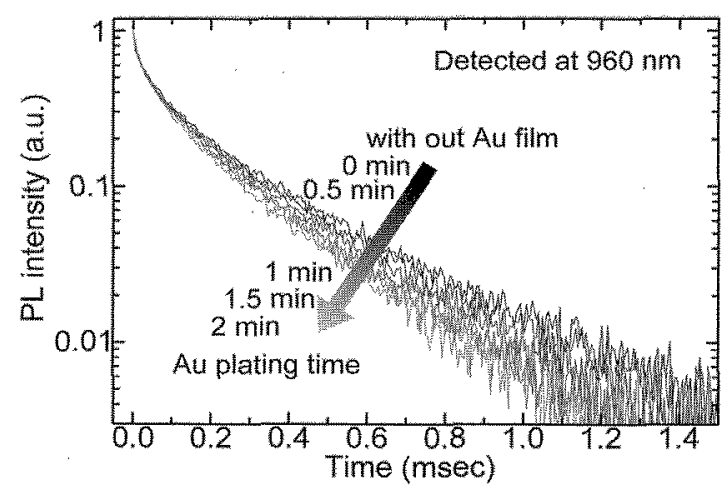

Fig. 4. PL decay curves of Si-nes with rough $\mathrm{Au}$ films and that without $\mathrm{Au}$ films. 


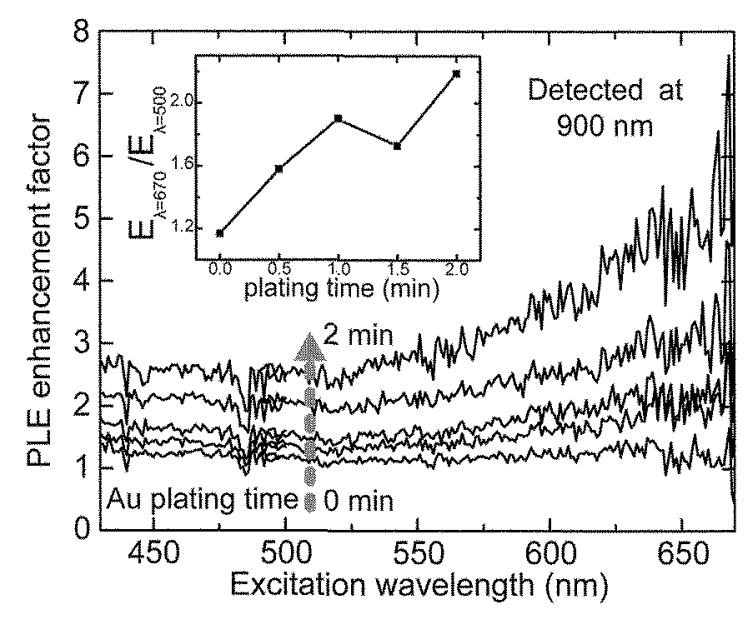

Fig. 5. PL intensity enhancement factors as a function of the excitation laser wavelength. Inset shows $E_{\lambda=670} / E_{\lambda=500}$, where $E_{\lambda=670}$ and $E_{\lambda=500}$ represent the enhancement factors excited at $670 \mathrm{~nm}$ and $500 \mathrm{~nm}$, respectively.

shortened by $\mathrm{Au}$ films and it becomes more significant at longer plating time. The shortening of the lifetime is considered to be due to the excitation of SPPs in a rough Au film.

Figure 5 shows the excitation wavelength dependence of enhancement factors obtained by dividing the PL intensity of the sample with a $\mathrm{Au}$ film by that of the sample without a Au film. The PL signals are detected at $900 \mathrm{~nm}$. Above $530 \mathrm{~nm}$, the enhancement factors increase, especially for the sample with longer plating time. About 5.5-fold enhancement is observed after 2 minute plating at $670 \mathrm{~nm}$. The wavelength corresponds to the surface plasmon resonance of rough Au films as shown in Fig. 2. This resonance behavior indicates that the PL intensity enhancement is related to the enhancement of an electric field of incident light due to the excitation of surface plasmons supported by the rough Au films.

The emission wavelength dependence of PL enhancement factors (Fig. 3(b)) and the enhanced decay rate (Fig. 4) indicate the radiative decay rate enhancement. Because of the excitation of SPPs, the PL lifetime of excitons confined in Si-ncs near a metal film can be expressed as

$$
\tau_{\text {metal }}=I /\left(W_{r}+W_{n r}+W_{s p p}\right)
$$

where $W_{r}$ and $W_{n r}$ represent the radiative and nonradiative decay rates of Si-ncs, respectively, and $W_{s p p}$ denotes the rate of nonradiative energy transfer from Si-ncs to SPPs. The observed shortening of PL lifetime is considered to be due to excitation of SPPs. On optically smooth metal surfaces, the excited SPPs modes do not couple with photons. Roughness and surface defects can scatter the SPPs to light [6]. If the excited SPPs are extracted with the efficiency of $\eta_{s p p}$, the PL quantum efficiency of Si-ncs is expressed as:

$$
\eta=\left(W_{r}+\eta_{s p p} W_{s p p}\right) /\left(W_{r}+W_{n r}+W_{s p p}\right)
$$

In the present work, the PL intensity is enhanced by rough Au films. This implies that excited SPPs are efficiently scattered by the roughness and $\eta_{s p p}$ is rather large.

If we compare Fig. 2 and $3(\mathrm{~b})$, we can see the correlation between the wavelengths of the absorption maxima and that of the enhancement factor except for the sample with the plating time of $2 \mathrm{~min}$. This indicates that PL is most efficiently enhanced near the SPPs resonance wavelength. In the case of the sample after 2 min plating, the wavelength of the enhancement factor maximum doesn't agree with that of the absorption maximum. One possible explanation is that localized SPPs become propagation type SPPs with increasing the $\mathrm{Au}$ film thickness, and the resonant frequency shifts to the bulk SPP frequency. The PL enhancement factors and the lifetime of Si-ncs depend on the degree of roughness of $\mathrm{Au}$ films as shown in Fig. 3(b) and Fig.4. This result suggests that $W_{s p p}$ and $\eta_{s p p}$ increase with increasing the degree of roughness.

The PL enhancements are caused not only by the increase of the decay rate of Si-ncs but also by the enhancement of an electric field of incident light. It is well known that the electromagnetic interaction between localized SPPs excited in metal nanostructures and optical waves results in the enhancement of the local fields. In fact, the PL enhancement factors are larger when the excitation wavelength corresponds to that of the surface plasmon resonance of rough Au films. It is also well known that the intensity of the local fields strongly depends on the size of metal nanostructures. Inset of Fig. 5 shows the ratios of the enhancement factors at $670 \mathrm{~nm}$ abd $500 \mathrm{~nm}\left(E_{\lambda=670} / E_{\lambda=500}\right)$. We can see that $E_{\lambda=670} / E_{\lambda=500}$ increases as the plating time increases. This result is consistent with theoretical prediction that local enhancement is larger at larger metal nanostructures in the present size range.

\section{CONCLUSIONS}

We demonstrated that the enhanced PL of Si-ncs can be achieved by placing it near rough $\mathrm{Au}$ films. We found that the PL intensity and the decay rate increases as the roughness becomes large. The PL excitation spectra revealed that the PL enhancement is the largest (about 5.5-hold) when the excitation wavelength corresponds to that of the SPPs of rough Au films. The PL enhancement is caused by the enhancement of an electric field of incident light due to the excitation of surface plasmons supported by the rough Au films and also the increase of radiative decay rate of Si-ncs. It is also revealed that these enhancement factors strongly depend on the degree of roughness.

[1] L. T. Canham, Appl. Phys. Lett., 57, 1046-48 (1990).

[2] C. Delerue, M. Lannoo, G. Allan, and E. Martin, Thin Solid Films, 255, 27-34 (1995)

[3] D. Kovalev, H. Hecker, G. Polisski, and F. Koch, Phys. Status Solidi B, 215, 871-932 (1999).

[4] S. Takeoka, M. Fujii, and S. Hayashi, Phys. Rev. B, 62, $16820-25(2000)$.

[5] I. Sychugov, R. Juhasz, J. Valenta, and J. Linnros, Phys. Rev. Lett., 94, 087405-08 (2005).

[6] K. Okamoto, I. Niki, A, Shvartser, Y. Narukawa, T. Mukai, and A. Scherer, Nat. Mater., 3, 601-05 (2004),

[7] H. Mertens, J. S. Biteen, H. A. Atwater, and A. Polman, Nano Lett., 6, 2622-25 (2006). 
[8] S. Pillai, K. R. Catchpole, T. Trupke, G. Zhang, J. Zhao, and M. A. Green, Appl. Phys. Lett., 88, 161102-04 (2006).

[9] Y. X. Zhang, K. Aslan, M. J. R. Previte, and C. D. Geddes, Appl. Phys. Lett., 90, 053107-09 (2007).

[10] J. S. Biteen, D. Pacifici, N. S. Lewis, and H. A. Atwater, Nano Lett., 5, 1768-73 (2005).

[11] E. Takeda, M. Fujii, T. Nakamura, Y. Mochizuki, and S. Hayashi, J. Appl. Phys., 102, 023506-11 (2007).

[12] Y. Kanzawa, T. Kageyama, S. Takeoka, M. Fujii, S. Hayashi, and K. Yamamoto, Solid State Commun., 102, 533-37 (1997).

[13] M. Fujii, A. Mimura, S. Hayashi, and K. Yamamoto, Appl. Phys. Lett., 75, 184-86 (1999).

(Received December 10, 2007;Accepted Febrary 8, 2008) 